\title{
EFECTOS DEL TRATAMIENTO ANTIOXIDANTE EN LA ACTIVACIÓN DEL EJE HIPOTÁLAMO-HIPÓFISO-ADRENAL INDUCIDA POR EL CONSUMO DE DIETAS RICAS EN SACAROSA
}

\author{
EFFECTS OF ANTIOXIDANT TREATMENT ON THE ACTIVATION \\ OF THE HIPOTALAMO- HIPOFISO-ADRENAL AXIS INDUCED \\ BY THE CONSUMPTION OF SUCROSE RICH DIETS
}

\author{
María Elisa Mercau ${ }^{1,2}$, Juan Salvador Calanni ${ }^{1,3}$, Marcos Aranda ${ }^{1,4}$, Ruth Estela Rosenstein ${ }^{1,5}$, \\ Esteban Martín Repetto ${ }^{1,6}$, Cora Beatriz Cymeryng ${ }^{1,7}$
}

\section{RESUMEN}

Introducción: dados los efectos pleiotrópicos de los glucocorticoides (GCs) sobre el metabolismo, los niveles excesivos y sostenidos de GCs circulantes tienen efectos deletéreos e incrementan la morbilidad y mortalidad cardiovascular.

Objetivos: estudiar el efecto de la terapia antioxidante (con ácido lipoico o melatonina) sobre la hiperactivación del eje hipotálamo-hipófiso-adrenal (HHA) en animales alimentados con dieta rica en sacarosa (DRS).

Materiales y métodos: se evaluó la actividad del eje HHA y se determinaron parámetros hormonales, de estrés oxidativo y de inflamación en la adenohipófisis de animales tratados con DRS durante tres semanas.

Resultados: los animales del grupo DRS mostraron mayores niveles circulantes de hormona adrenocorticotropa (ACTH, por sus siglas en inglés) y corticosterona. En paralelo se detectó un aumento en la expresión del polipéptido precursor (proopiomelanocortina, POMC) y de ACTH en la adenohipófisis, donde también se observó un aumento de lipoperóxidos y proteínas nitradas en tirosina (daño oxidativo), un mayor número de macrófagos tisulares y un incremento en la producción de IL-1beta. El tratamiento antioxidante previno los cambios en estos parámetros. En particular la melatonina también normalizó la actividad del eje HHA y la expresión hipofisaria de POMC.

Conclusiones: la sobrecarga metabólica inducida por la administración de DRS genera daño oxidativo e inflamación en la adenohipófisis. La activación de los macrófagos tisulares producida en consecuencia podría impactar sobre los corticotropos hipofisarios e inducir su hiperfunción. La melatonina podría utilizarse como herramienta terapéutica para normalizar la actividad del eje HHA en modelos de obesidad por dieta.

Palabras clave: dieta rica en sacarosa; adenohipófisis; eje hipotálamo-hipófiso-adrenal; estrés oxidativo; inflamación; ácido lipoico; melatonina.

Revista de la Sociedad Argentina de Diabetes 2020; Vol. 54 (39-51)

\section{ABSTRACT}

Introduction: given the pleiotropic effects of glucocorticoids (GCs) on metabolism, excessive and sustained levels of circulating GCs, have deleterious effects and increase cardiovascular morbidity and mortality.

Objectives: to study the effect of antioxidant therapy on hyperactivation of the hypothalamic-pituitary-adrenal (HPA) axis in animals fed a sucrose-rich diet (SRD).

Materials and methods: the activity of the HPA axis was evaluated and hormonal, oxidative stress and inflammation parameters were determined in the adenohypophysis of animals treated with SRD for trhee weeks.

Results: animals from the SRD group showed higher circulating levels of adrenocorticotropic hormone (ACTH) and corticosterone. In parallel, an increase in the expression of the polypeptide precursors, POMC and ACTH were detected in the adenohypophysis. We also observed an increase in lipoperoxides and proteins nitrated in tyrosine (oxidative damage), a greater number of tissue macrophages and an increase in the production of IL-1beta. Antioxidant treatment prevented all these changes. In particular, melatonin also normalized the activity of the HPA axis and pituitary expression of POMC.

Conclusions: the metabolic overload induced by the administration of SRD generates oxidative damage and inflammation in the adenohypophysis. Activation of tissue macrophages could affect, in turn, pituitary corticotrophs inducing their activation. Melatonin could be used as a therapeutic tool to normalize the activity of the HPA axis in diet obesity models.

Key words: sucrose rich diet; adenohypophysis; hypothalamicpituitary-adrenal axis; oxidative stress; inflammation; lipoic acid; melatonin.

Revista de la Sociedad Argentina de Diabetes 2020; Vol. 54 (39-51) 
1 Facultad de Medicina, Departamento de Bioquímica Humana, Universidad de Buenos Aires, Centro de Estudios Farmacológicos y Botánicos-Consejo Nacional de Investigaciones Científicas y Técnicas (CEFYBO-CONICET), Ciudad Autónoma de Buenos Aires, Argentina

2 Doctora en Ciencias Biológicas, Post-doctorando en el Departamento de Inmunobiología, Universidad de Yale, New Haven, Connecticut, Estados Unidos

3 Lic. en Ciencias Biológicas, Tesista en el Departamento de Bioquímica Humana, Universidad de Buenos Aires, Centro de Estudios Farmacológicos y Botánicos-Consejo Nacional de Investigaciones Científicas y Técnicas (CEFYBO-CONICET), Ciudad Autónoma de Buenos Aires, Argentina

4 Doctor en Ciencias Biológicas, Post-doctorando en la Universidad Northwestern, Chicago, Estados Unidos

5 Doctora en Ciencias Químicas, Investigadora Superior del Consejo Nacional de Investigaciones Científicas y Técnicas (CONICET), Profesora Titular del Departamento de Bioquímica Humana de la Facultad de Medicina, Universidad de Buenos Aires, Ciudad Autónoma de Buenos Aires, Argentina
6 Doctor en Bioquímica, Investigador Adjunto del Consejo Nacional de Investigaciones Científicas y Técnicas (CONICET), Jefe de Trabajos Prácticos en el Departamento de Bioquímica Clínica de la Facultad de Bioquímica, Universidad de Buenos Aires, Ciudad Autónoma de Buenos Aires, Argentina

Doctora en Ciencias Químicas, Investigadora Principal del Consejo Nacional de Investigaciones Científicas y Técnicas (CONICET), Profesora Adjunta en el Departamento de Bioquímica Humana de la Facultad de Medicina, Universidad de Buenos Aires, Ciudad Autónoma de Buenos Aires, Argentina

Contacto de la autora: Cora Beatriz Cymeryng

E-mail: cymeryng@fmed.uba.ar Correspondencia: Paraguay $21555^{\circ}$ (C1121ABG), Ciudad Autónoma de Buenos Aires, Argentina

Fecha de trabajo recibido: 21/01/20

Fecha de trabajo aceptado: 24/04/20

Conflictos de interés: los autores declaran que no existe conflicto de interés

\section{INTRODUCCIÓN}

El síndrome metabólico (SM) se caracteriza por la presencia de obesidad, dislipidemia, hipertensión, hiperinsulinemia e hiperglucemia leve'. Entre otras complicaciones a largo plazo, el establecimiento de insulinorresistencia (IR) es un factor de riesgo para las enfermedades cardiovasculares y la diabetes mellitus tipo $2(\mathrm{DM} 2)$ y, por lo tanto, el desarrollo de prácticas de prevención es un objetivo relevante para la Salud Pública.

La prevalencia creciente del SM se asocia, entre otros factores, a cambios en el estilo de vida y el consumo de dietas inadecuadas ${ }^{2}$. En cuanto a esto último, diversos estudios indicaron que la ingesta de un exceso de fructosa o sacarosa puede generar $S^{3-5} y$, en ese sentido, la alimentación de roedores con dietas ricas en sacarosa (DRS) demostró ser un modelo útil para evaluar los mecanismos involucrados porque presenta características similares a las observadas en pacientes con SM ${ }^{6-8}$.

Se ha postulado que un incremento en los niveles circulantes de glucocorticoides (o de su activación en tejidos blanco) participaría del desarrollo y mantenimiento de la obesidad y del SM, ya que pacientes con síndrome de Cushing, por ejemplo, presentan un metabolismo anormal de lípidos y glucosa que se acompaña de $I^{9-12}$. Sin embargo, el análisis de los estudios publicados no arroja resultados concluyentes ${ }^{13-15}$.

Recientemente demostramos que ratas alimentadas con sacarosa al $30 \%$ en el agua de bebida (DRS) desarrollan IR y cambios en la actividad del eje hipotálamo-hipófiso- adrenal $(\mathrm{HHA})^{16,17}$.
Luego de siete semanas de tratamiento, detectamos un aumento en los niveles circulantes de glucocorticoides (GCs), que correlacionó con una respuesta alterada a la insulina ${ }^{18}$.

El desarrollo de IR se relacionó con la generación de estrés oxidativo, la producción de citoquinas proinflamatorias, la inducción de una respuesta de fase aguda y la activación de los macrófagos del tejido adiposo ${ }^{19-21}$. De hecho, se ha postulado que la producción de especies reactivas de oxígeno (ROS, por sus siglas en inglés) es una vía común para la inducción de IR por glucosa, ácidos grasos libres y citoquinas ${ }^{22}$.

Tanto las citoquinas como las ROS pueden afectar la actividad del eje $\mathrm{HHA}^{23-28}$. En particular, la producción de hormona adrenocorticotropa $(\mathrm{ACTH}$, por sus siglas en inglés) por células corticotropas hipofisarias se estimula en presencia de citoquinas y en concentraciones suprafisiológicas de glucosa (que implican la generación de ROS) ${ }^{29,30}$.

Diversos estudios demostraron los efectos beneficiosos de la suplementación dietaria con micronutrientes con propiedades antioxidantes, como el ácido $\alpha$-lipoico (o tióctico -AL-), como complemento a las terapias clásicas para la prevención o tratamiento de complicaciones diabéticas como la neuropatía, nefropatía y cardiomiopatías diabéticas $^{31,32}$. El AL es un ditio-compuesto que participa del metabolismo energético mitocondrial y junto con su metabolito, el ácido dihidrolipoico, son considerados buenos antioxidantes porque son potentes eliminadores de ROS y de especies 
reactivas del nitrógeno (RNS, por sus siglas en inglés), en dominios lipídicos y acuosos son quelantes de metales ${ }^{33}$, y están implicados en la regeneración de otros antioxidantes como las vitaminas E y C, y el glutatión reducido ${ }^{34}$.

La melatonina (5 metoxi-N-acetiltriptamina), producida por la glándula pineal, es una molécula multifuncional que participa, entre otros procesos, de la regulación del ritmo circadiano, la respuesta inmune y el período fértil en animales con ciclos reproductivos ${ }^{35,36}$. La melatonina es, además, un importante depurador de ROS y RNS, tanto en concentraciones fisiológicas como farmacológicas ${ }^{37,38}$, activa enzimas antioxidantes como la superóxido dismutasa (SOD) y la glutatión peroxidasa (GSH-Px) e induce la actividad de la $\gamma$-glutamilcisteína sintetasa al estimular así la producción de glutatión $(\mathrm{GSH})^{39,40}$. Asimismo múltiples evidencias experimentales demuestran propiedades antiinflamatorias de la melatonina relacionadas con la inhibición de la óxido nítrico sintasa inducible iNOS (sus siglas en inglés) y la eliminación del peroxinitrito ${ }^{41,42}$.

En nuestro laboratorio, en este modelo animal de IR, detectamos un incremento en la actividad del eje HHA que fue previo a cambios en la sensibilidad a insulina. En ese sentido, en este estudio nos propusimos evaluar los efectos del tratamiento antioxidante (AL y/o melatonina) sobre la activación del eje HHA en animales alimentados con DRS. Nuestros resultados muestran los efectos beneficiosos del tratamiento con ambas moléculas sobre los cambios observados en la función hipofisaria en ratas alimentadas con DRS, y sugieren que el estrés oxidativo y la inflamación determinadas a nivel hipofisario participan de la desregulación del eje HHA.

\section{MATERIALES Y MÉTODOS Animales}

En este estudio se utilizaron ratas Wistar macho adultas (200-250 g) alojadas (tres animales/ jaula) en condiciones controladas de humedad y temperatura $\left(21 \pm 2^{\circ} \mathrm{C}\right)$, con un ciclo de luz-oscuridad de 12 horas (h). Los animales recibieron alimento estándar ad libitum y como bebida, agua (grupo C) o una solución de sacarosa al 30\% p/v (grupo DRS) durante tres semanas. Se evaluó la resistencia a la insulina mediante una prueba de tolerancia a la insulina durante la tercera semana de tratamiento ${ }^{12}$. Todos los protocolos fueron aprobados por el Comité de Uso y Cuidado de Animales (CICUAL) de la Facultad de Medicina de la Universidad de Buenos Aires y siguieron los "Principios del cuidado de animales de laboratorio" (publicación NIH No 85-23, revisada en 1985).

\section{Tratamientos antioxidantes}

Los animales se dividieron al azar en cuatro grupos experimentales: C (control), DRS, antioxidante y antioxidante con DRS. Los tratamientos con antioxidantes y DRS se iniciaron simultáneamente.

Se utilizaron dos antioxidantes diferentes: ácido lipoico ( $A L$ ) y melatonina (MEL). Los animales recibieron $A L, 100 \mathrm{mg} / \mathrm{kg}$, i.p., cada 48 h. Los animales de los grupos sin tratamiento con AL recibieron inyecciones de solución salina con la misma frecuencia como tratamiento simulado. A otro grupo de animales se le implantó un pellet subcutáneo de melatonina $(20 \mathrm{mg}$ con $3 \%$ p/v de aceite vegetal, comprimido en un cilindro de $2,5 \mathrm{~mm}$ de diámetro y $1 \mathrm{~mm}$ de longitud), mientras que los grupos controles fueron sometidos a una operación simulada sin implante de pellet. La melatonina se obtuvo de Sigma Chemical Co. (St. Louis, MO, EE.UU.). El pellet de melatonina se implantó subcutáneamente debajo de la piel del cuello y se reemplazó cada 10 días ${ }^{43}$.

\section{Muestras de tejido y suero}

Finalizados los tratamientos, los animales se sacrificaron por decapitación, entre las 9:00 h y las 10:00 h para minimizar las variaciones circadianas entre los grupos, y se recolectó sangre troncal. Las glándulas hipofisarias se disecaron sobre hielo y se homogenizaron en buffer fosfato $(\mathrm{Na} 2 \mathrm{H}$ PO4 50 mmol/L pH 7.4; EDTA 0.2 mmol/L, KCl 100 $\mathrm{mmol} / \mathrm{L}$ ) con inhibidores de proteasas (cocktail 1X, Sigma Aldrich, Argentina) o en reactivo TRI (Genbiotech, Argentina) para el aislamiento de ácido ribonucleico (ARN) total.

\section{Mediciones bioquímicas y hormonales}

Los esteroides se extrajeron del suero con diclorometano y su concentración sérica se determinó por radioinmunoensayo, como se describió anteriormente ${ }^{44}$. La concentración plasmática de ACTH se determinó mediante el sistema de inmunoensayo Immulite $2000^{\circledR}$ (Siemens, Alemania), y los niveles séricos de glucosa y triglicéridos se evaluaron mediante ensayos comerciales colorimétricos (Wiener Lab, Argentina o Randox, Reino 
Unido). La concentración de melatonina en suero se analizó mediante un ensayo de ELISA competitivo (IBL International INC, Hamburgo, Alemania).

\section{Niveles de lipoperóxidos y actividad de catalasa}

Los tejidos hipofisarios se homogenizaron en $400 \mu \mathrm{l}$ de bufferTBARS (KH2PO4 15 mM/ K2PO4, $\mathrm{KCl} 60 \mathrm{mM}, \mathrm{pH} 7,4)$ con inhibidores de proteasas. Los niveles de lipoperóxidos se determinaron en los sobrenadantes de centrifugación (1.000 xg por 10 minutos a $4^{\circ} \mathrm{C}$ ), como especies reactivas al ácido tiobarbitúrico, de acuerdo a protocolos ya descriptos ${ }^{45}$.

La actividad de la catalasa se determinó según los procedimientos publicados ${ }^{46}$ con modificaciones menores. Brevemente, los homogenatos hipofisarios se diluyeron 1:10 en buffer de fosfato sódico 50 mM, pH 7.4 y se colocaron $100 \mu \mathrm{l}$ de esta dilución en una cubeta espectrofotométrica. Las reacciones se iniciaron mediante la adición de $60 \mu \mathrm{l}$ de $\mathrm{H}_{2} \mathrm{O}_{2} 2.5$ volúmenes ( $223 \mathrm{mM}$ ) y se registró la absorbancia a $240 \mathrm{~nm}$ durante 120 segundos. La actividad, expresada como $\mu \mathrm{M}$ de $\mathrm{H}_{2} \mathrm{O}_{2}$ consumido por minuto por $\mu \mathrm{g}$ de proteína, se calculó a partir de la pendiente de la curva correspondiente considerando un coeficiente de absorción molar de $0.0394 \mathrm{mM}^{-1} \mathrm{~cm}^{-1}$ para el $\mathrm{H}_{2} \mathrm{O}_{2}$.

\section{Inmunohistoquímica}

Los animales se anestesiaron con una mezcla de ketamina y xilazina (50 mg/kg-2 mg/ $/ \mathrm{kg}$ ) y luego se perfundieron intracardíacamente con paraformaldehído al $4 \%$ en buffer fosfato $0.1 \mathrm{M}, \mathrm{pH}$ 7.4. Los tejidos hipofisarios disecados se fijaron posteriormente en el mismo fijador (12 h) y se deshidrataron incubándolos con soluciones de concentraciones crecientes de etanol (70\%, 90\% y $100 \% \mathrm{v} / \mathrm{v})$. Los tejidos se clarificaron con acetato de N-butilo y se incluyeron en parafina (Biopack, CABA, Argentina). Las glándulas hipofisarias se cortaron en secciones de $4 \mu \mathrm{m}$ usando un micrótomo giratorio (RM2125 RTS, Leica, Alemania). Cada sección se recolectó en portaobjetos cargados y luego se deshidrataron por calor $\left(50^{\circ} \mathrm{C}\right.$ durante 10 minutos). Luego de la desparafinación y la rehidratación, se realizó la recuperación del antígeno incubando las secciones en buffer citrato (citrato de sodio 10 mM, Tween-20 0,05\%, pH 6.3) durante 30 minutos a $100^{\circ} \mathrm{C}$. El tejido se permeabilizó con Tritón X-100 0,3\% en PBS 1X. Después de tres lavados, los cortes se incubaron durante $1 \mathrm{~h}$ en solución de bloqueo ( $2 \%$ de suero de caballo normal). La inmunodetección se realizó utilizando los siguientes anticuerpos primarios: anti-F4/80 (1:1000, Santa Cruz Biotechnology, EE.UU.) o anti-IBA-1 (1:500, Wako Laboratory, EE.UU.) y anticuerpos secundarios conjugados con fluoróforo (Jackson Immuno Research, EE.UU.). Los núcleos se tiñeron con DAPI y los cortes se montaron con medios para fluorescencia (Vectashield, Vector Laboratories, EE.UU.), y las imágenes se adquirieron en un microscopio de fluorescencia (BX-50 Olympus, EE. UU.) a través de una cámara digital incorporada (3CCD, Sony, EE.UU.). El procesamiento se realizó con el software Image $\mathrm{J}(\mathrm{NIH}$, Bethesda, MD, EE.UU.). El número de células positivas para Iba1 se contó en un área de 500 x 500 píxeles (12 imágenes por tratamiento).

\section{Ensayos de PCR en tiempo real (qRT-PCR)}

La transcripción inversa se realizó con la enzima MMLV (Life Technologies, Argentina) como se describió anteriormente ${ }^{47}$. Las amplificaciones por PCR en tiempo real se efectuaron en un termociclador Rotor-Gene Corbett Life Science (Corbett Research, Sidney, NSW, Australia) y para la cuantificación se utilizó el software Rotor Gene 6000 Series (versión 1.7 Build 40, Hilden, Alemania). Las secuencias de los primeros utilizados en este estudio se muestran en la Tabla 1. Los niveles de expresión génica se normalizaron con Actb (actina) como control interno con el método de cuantificación relativa de $\Delta \Delta \mathrm{Ct}^{48}$.

\section{Análisis por inmunoblot}

Las muestras de tejido se sometieron a SDSPAGE y transferencia a membranas de PVDF con el sistema Trans-Blot Semi-Dry (Bio-Rad Laboratories Inc., EE.UU.). Las membranas se trataron como se describió anteriormente ${ }^{13}$. Para los inmunoblots de POMC/ACTH las muestras se analizaron por electroforesis en geles de poliacrilamida con SDS con algunas modificaciones al protocolo estándar. Brevemente se utilizaron geles separadores de acrilamida/bisacrilamida al $16 \%$ preparados en Tris- $\mathrm{HCl} 1 \mathrm{M} \mathrm{pH} 8.45$, SDS 0,3\%, glicerina $10 \%$ y los buffers de corrida fueron para el buffer del ánodo: Tris-HCl 100 mM pH 8.9 y para el del cátodo Tris-Tricina $100 \mathrm{mM}$, SDS 0,1\% pH 8.25. Las proteínas se transfirieron a membranas de PVDF a $15 \mathrm{~V}$, overnight, en tris-acetato $300 \mathrm{mM} \mathrm{pH} \mathrm{8.6.}$ Para el revelado del inmunoblot se utilizó el an- 
ticuerpo monoclonal de ratón contra ACTH (Santa Cruz Biotechnology Cat. No sc-52980, RRID: AB_831670).

Luego de la incubación con un segundo anticuerpo adecuado (IgG de caballo, anti-ratón conjugado con peroxidasa de rábano picante, Cell Signaling Technology, EE.UU., Cat N ${ }^{\circ}$ 7076, RRID: AB_330924), las membranas se revelaron con el reactivo de ECL (GE, EE.UU.), la quimioluminiscencia se detectó con el sistema Image Quant Imaging (GE Healthcare, Pittsburgh, EE.UU.) y la cuantificación se realizó con el software Image J (NIH, Bethesda, MD, EE.UU.).

\section{Análisis estadístico}

Todos los valores se expresaron como media \pm SEM de $n$ determinaciones experimentales. Las diferencias entre los grupos se analizaron mediante la prueba t de Student no pareada de dos colas o por ANOVA de una vía, según correspondiera. Cuando el ANOVA arrojó diferencias significativas $(p<0,05)$, se efectuaron comparaciones post hoc (prueba de Tukey o de Dunnett) para determinar la diferencia estadística entre los grupos. Para todos los cálculos se usó GraphPad InStat versión 3.06 para Windows (GraphPad Software, San Diego, CA).

\begin{tabular}{|l|c|}
\hline & MEL $(\mathrm{pg} / \mathrm{ml})$ \\
\hline Sin tratamiento & $153,2 \pm 49,4$ \\
Mel (implante con Mel-3d) & $951,7 \pm 87,9 * *$ \\
Mel (implante con Mel-7d) & $466,3 \pm 87,0 *$ \\
\hline
\end{tabular}

Los datos se expresan como media \pm SEM, $n=4$. ${ }^{*} p<0,05 y{ }^{* *} p<0,01$ vs. control sin tratamiento por ANOVA seguido del test de Dunnett.

Tabla 1: Niveles de melatonina circulante en animales sin y con tratamiento con melatonina.

\section{RESULTADOS}

El consumo de DRS durante tres semanas indujo incrementos significativos en los parámetros metabólicos, como glucemia y trigliceridemia, determinados luego de $6 \mathrm{~h}$ de ayuno (GLU, C: $93,3 \pm 1,9$ vs. DRS: $102,3 \pm 1,9, p<0,05$; TAG, C: $75,5 \pm 4,4$ vs. DRS: $101,0 \pm 8,8, p<0,001)$. En esta etapa del tratamiento no se observaron diferencias significativas en el peso corporal (C: $304,0 \pm 10,5$ vs. DRS: $331,7 \pm 7,9, p=0,27$ ) o en la sensibilidad a la insulina entre ambos grupos de animales (KITT C: $3,33 \pm 0,34$ vs. DRS: $3,32 \pm 0,30, p=0,98)$.

Los animales tratados con DRS también presen- taron mayores niveles circulantes de ACTH y corticosterona que los correspondientes controles (Figura 1A). Estos resultados sugieren que el consumo de DRS induce cambios en la actividad del eje HHA que son previos a los cambios en la sensibilidad a la insulina. En concordancia, en la adenohipófisis de estos animales se determinó un incremento en los niveles de ARNm del polipéptido precursor POMC y del peptido ACTH (Figura 1B y C).

Con el fin de analizar los procesos involucrados en los cambios hormonales observados, se evaluó el efecto de la dieta sobre la generación de estrés oxidativo/nitrosativo e inflamación en la adenohipófisis. Nuestros resultados indicaron un aumento significativo en los niveles de lipoperóxidos (medidos como sustancias reactivas al ácido tiobarbitúrico o TBARS, Figura 2A) y de proteínas modificadas con nitrotirosina (Figura 2B) en los animales del grupo DRS. Con respecto a las enzimas antioxidantes, detectamos una mayor actividad de catalasa (Figura 2C) y una mayor expresión de hemoxigenasa-1 (HO-1) (Figura 2D) en las adenohipófisis de estos animales. Asimismo observamos un número significativamente mayor de células positivas para el marcador de macrófagos $(F 4 / 80+)$ y para el de células derivadas de monocitos (IBA-1 $1^{+}$) (Figura 3D), y un aumento en los niveles de la proteína de F4/80 (Figura 2F) en la adenohipófisis de los animales del grupo DRS.

En conjunto, nuestros resultados sugirieron que la alimentación con DRS durante tres semanas indujo estrés oxidativo/nitrosativo e inflamación, en paralelo con un aumento significativo en los niveles de POMC y ACTH en el tejido adenohipofisario. Analizamos, entonces, el efecto del tratamiento antioxidante administrado desde el inicio de la modificación dietaria sobre los parámetros mencionados anteriormente.

Ninguno de los antioxidantes utilizados tuvo efecto sobre el peso corporal ni sobre los niveles séricos de TAG o glucosa en los grupos estudiados (datos no mostrados). Los resultados indicaron que la melatonina, pero no el ácido lipoico, impidió eficazmente la generación de peróxidos lipídicos en el tejido adenohipofisario (Figura 3A). Ambos antioxidantes previnieron, en cambio, el incremento de la actividad de catalasa (Figura 3B) y la expresión de hemoxigenasa-1 (Figura 3C), y también evitaron el aumento de la inmunorreactividad para IBA-1 (Figura 3D) en las glándulas pituitarias de las ratas tratadas con DRS. Los tratamientos antioxi- 
dantes previnieron, asimismo, el incremento en los niveles de expresión de TNF- $\alpha$ o de IL-1 $\beta$.

Finalmente evaluamos el efecto de los antioxidantes sobre la actividad del eje HHA. El tratamiento con melatonina previno la hiperactivación de los corticotropos hipofisarios en ratas tratadas con DRS, como lo demuestran la expresión hipofisaria del péptido ACTH (Figura 4A), y los niveles circulantes de ACTH (Figura 4B) y corticosterona (Figura
4C) en el grupo DRS+MEL. El ácido lipoico per se tuvo un importante efecto estimulatorio sobre la producción de ACTH hipofisaria (y también sobre la corticosteronemia) y no previno el incremento en ambos parámetros en los animales del grupo DRS (Figuras 4B y C). Estos resultados sugieren la posible utilización de melatonina en la prevención de las alteraciones tempranas en la actividad del eje HHA inducidas por dietas ricas en sacarosa.
A)

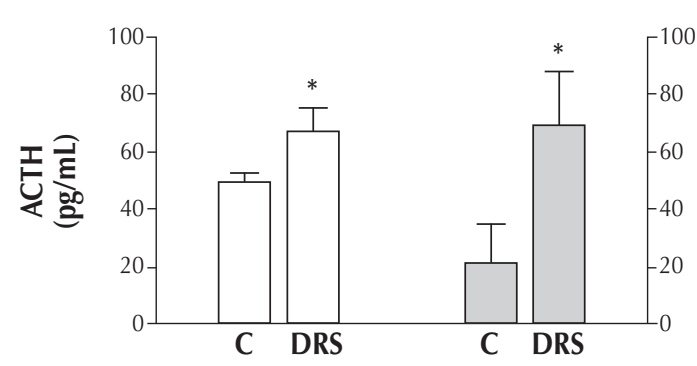

B)

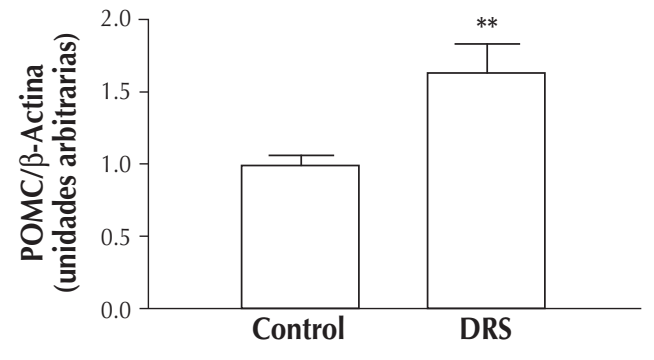

C)
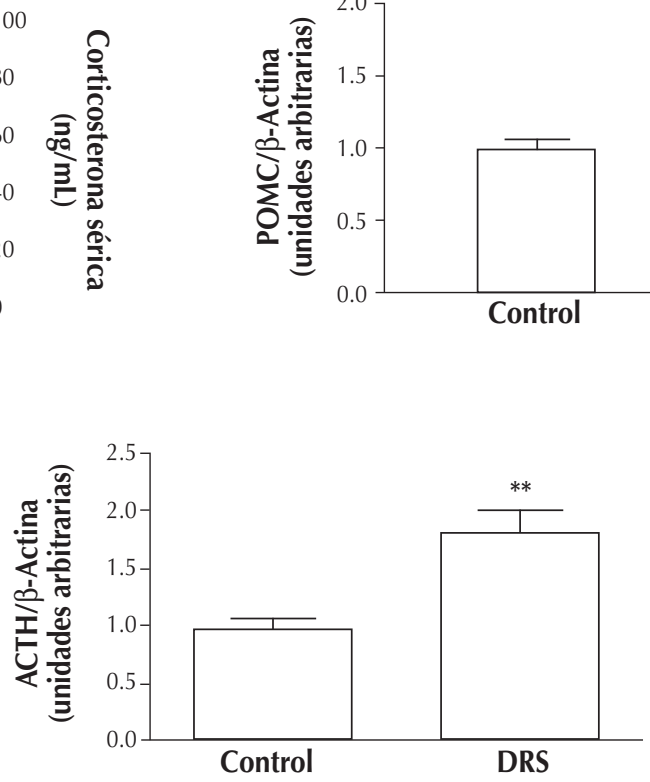

Se analizaron los niveles circulantes de ACTH y corticosterona por un inmunoensayo quimioluminiscente y por RIA respectivamente (A). Los niveles de ARNm de POMC se determinaron por RT-qPCR (B). Los datos se muestran como media $\pm S E M, n=12$ animales por grupo, ${ }^{*} p<0,05 y *{ }^{*} p<0,01$ vs. control por test $t$ de Student. C) Se muestra un inmunoblot representativo de la expresión de ACTH en la adenohipófisis y el análisis densitométrico correspondiente a cuatro experimentos independientes. Los datos se muestran como media $\pm S E M$, $n=4$ animales por grupo, ${ }^{*} p<0,01$ vs. control por test $t$ de Student.

Figura 1: Activación del eje HHA por la administración de una DRS durante tres semanas. 
A)

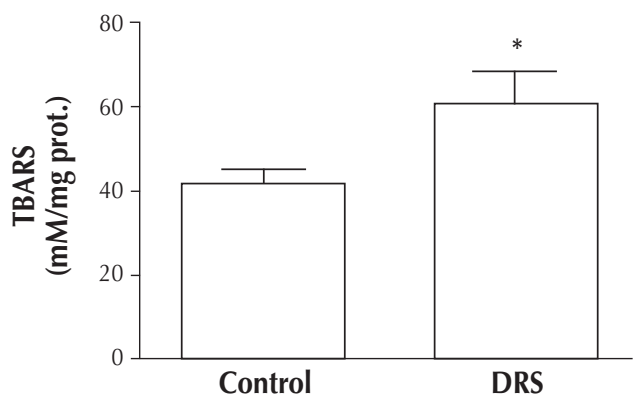

C)

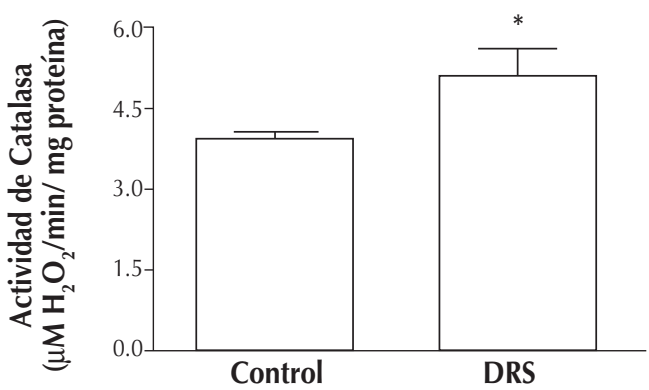

E)
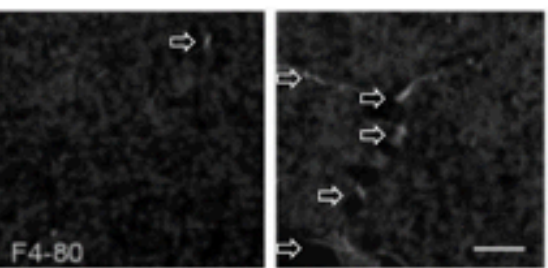

Control
B)

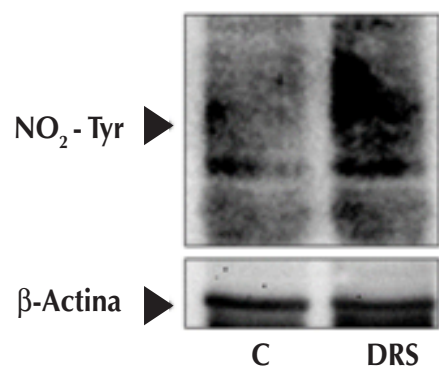

D)

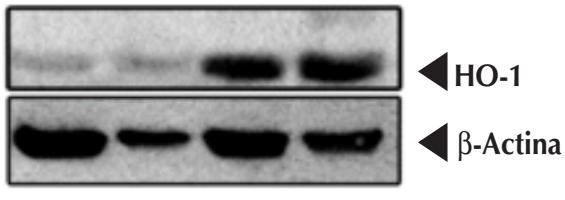

Control DRS

F)

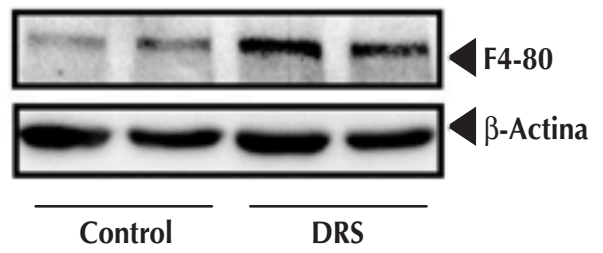

A) Niveles de lipoperóxidos determinados como TBARS. Los datos se muestran como media $\pm S E M, n=12,{ }^{*} p<0,05$ vs. $C$ por el test $t$ de Student. En B) se muestra un inmunoblot representativo de los niveles de proteínas modificadas en nitro-tirosina. C) Actividad de catalasa expresada como media $\pm S E M, n=6{ }^{*} p<0,05$ vs. C por el test $t$ de Student. En D) se muestra un inmunoblot representativo con los niveles de expresión de HO-1. E) Evaluación inmunohistoquímica de señal positiva para el marcador de células derivadas de monocitos, F4/80 (flechas blancas). Los núcleos se tiñeron con DAPI (gris oscuro). Barra de escala: 75 micrones. F) Inmunoblot representativo de los niveles de F4/80 en la adenohipófisis.

Figura 2: Estrés oxidativo e inflamación en la adenohipófisis de animales tratados con DRS durante tres semanas. 
A)

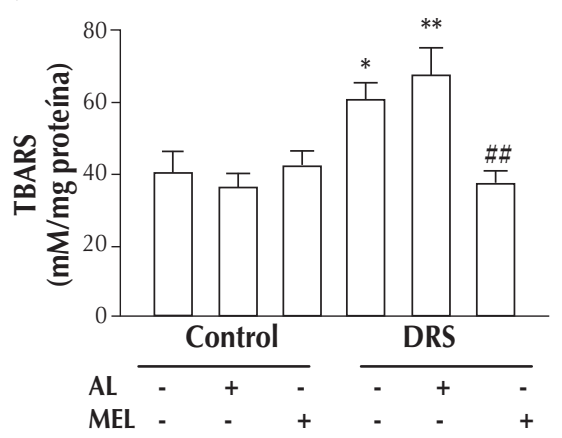

C)

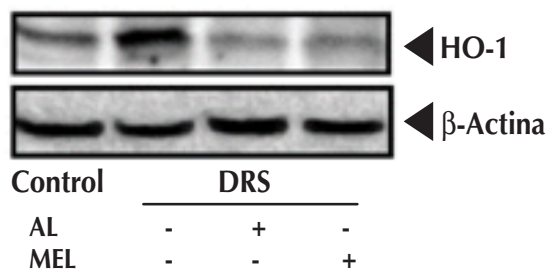

D)
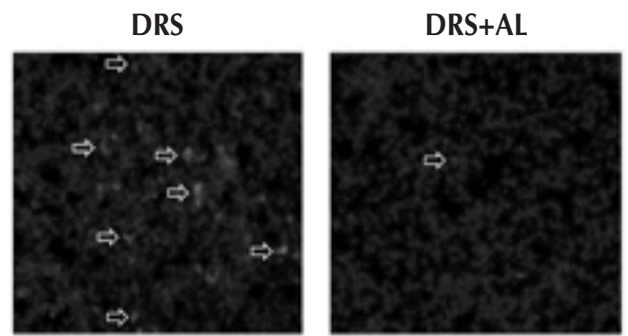

B)

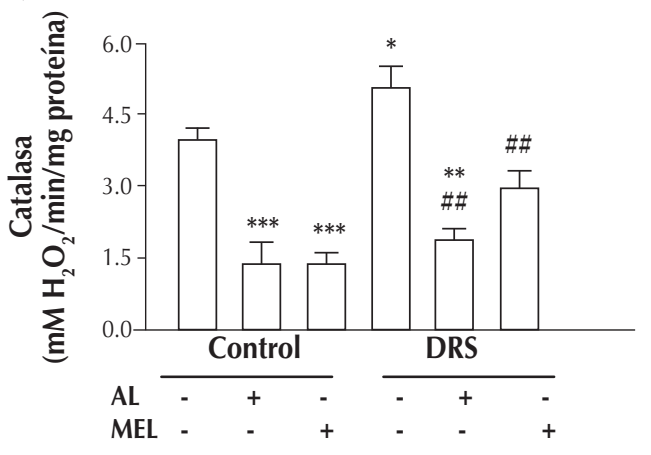

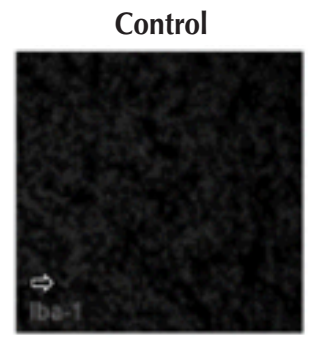

Control

E)

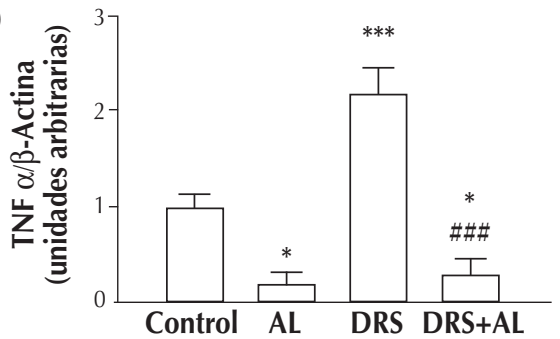

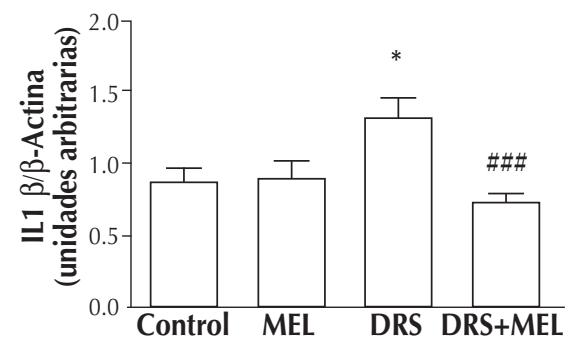

A) Niveles de lipoperóxidos evaluados como TBARS. B) Actividad de catalasa. Los datos (A y B) se muestran como media $\pm S E M, n=10$, ${ }^{*} p<0,05$ vs. control por ANOVA seguido del test de Tukey. C) Se muestra un inmunoblot representativo de la expresión de HO-1. D) Evaluación inmunohistoquímica de señal positiva para el marcador de células derivadas de monocitos, Iba-1 (flechas blancas). Los núcleos se tiñeron con DAPI (gris oscuro). Barra de escala: 75 micrones. E) Niveles de ARNm de IL 1- $\beta$ y de TNF- $\alpha$ normalizados con los de $\beta$-actina se determinaron por RT-qPCR. Los valores se indican como media \pm SEM. $n=4 ;{ }^{*} p<0,05 ;{ }^{* *} p<0,001 \mathrm{vs}$. control; $y^{\# \# *} p<0,001 \mathrm{vs}$. DRS, por ANOVA y test de Tukey.

Figura 3: El tratamiento antioxidante previene cambios en los parámetros de estrés oxidativo e inflamación en la adenohipófisis de ratas tratadas con DRS durante tres semanas. 
A)
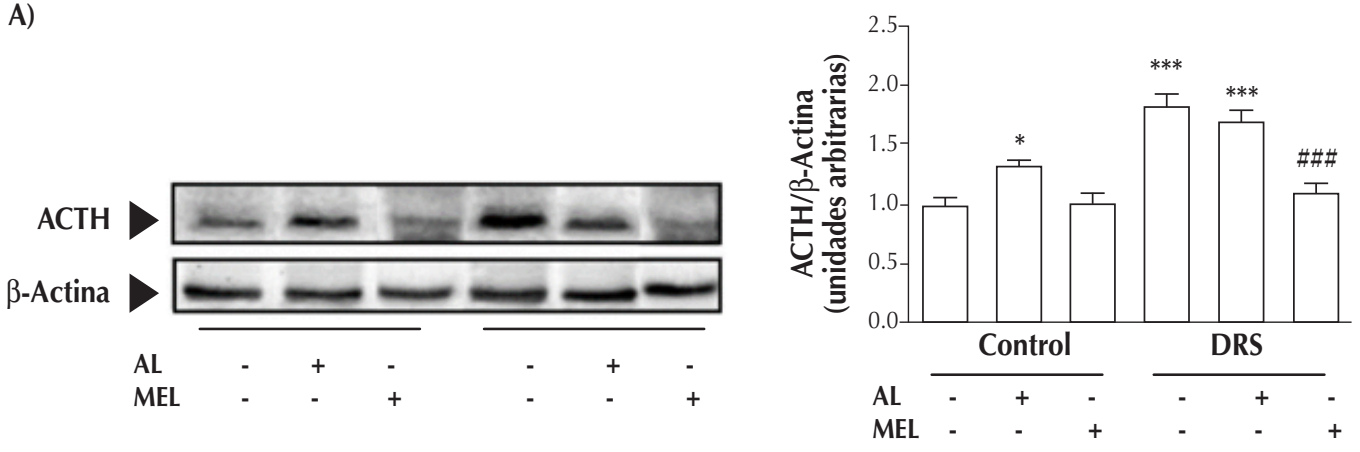

B)

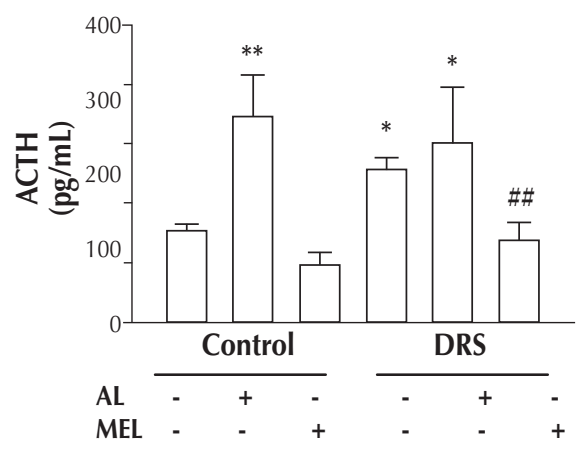

C)

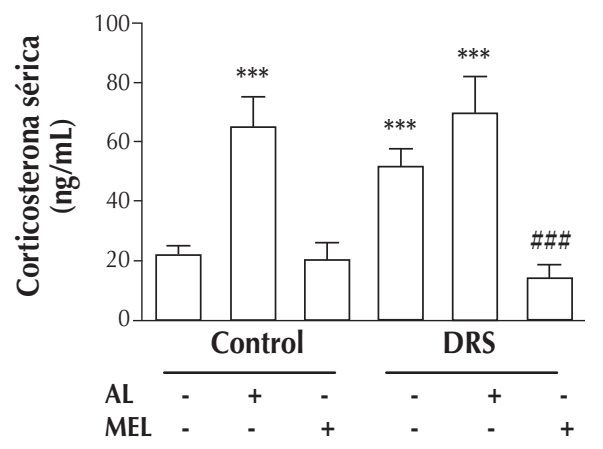

A) Inmunoblot representativo de la expresión hipofisaria de ACTH en animales tratados con DRS, AL y Mel, y la densitometría correspondiente a tres experimentos independientes. Los valores se indican como media $\pm S E M, n=3,{ }^{*} p<0,05,{ }^{* * *} p<0,001 \mathrm{vs}$. control, y ${ }^{\# \#} p<0,001$ vs. DRS, por ANOVA y test de Tukey. B) Concentración plasmática de ACTH determinada por un inmunoensayo quimioluminiscente. C) Niveles séricos de corticosterona evaluados por RIA. Los datos se muestran como media $\pm S E M . n=6 ;{ }^{*} p<0,05 ;{ }^{*} p<0,01$; ${ }^{* * *} p<0,001$ vs. control; $y{ }^{\#} p<0,01, " \# p<0,001$ vs. DRS, por ANOVA seguido de test de Tukey.

Figura 4: Efectos de la terapia antioxidante sobre la actividad del eje HHA.

\section{DISCUSIÓNY CONCLUSIONES}

Los resultados de este estudio demuestran la hiperactivación temprana del eje HHA en animales alimentados con DRS, que se evidencia por un aumento en los niveles circulantes de ACTH y corticosterona. Estos cambios se acompañaron de mayores niveles adenohipofisarios del precursor POMC y de ACTH, y por la aparición del estrés oxidativo e inflamación en el tejido. El tratamiento antioxidante evitó los cambios en los parámetros de estrés oxidativo e inflamación y, en particular, la administración de melatonina también normalizó la actividad del eje HHA.

Diversos estudios demostraron que una mayor disponibilidad de nutrientes incrementa la producción tisular de especies reactivas de oxígeno/ni- trógeno (ROS/RNS) al sobrepasar la capacidad antioxidante del tejido y producir daño oxidativo ${ }^{49-51}$.

Dado que en estos animales observamos mayores niveles circulantes de nutrientes, planteamos la hipótesis de que la disfunción hipofisaria observada se produce como consecuencia de la generación de estrés oxidativo a nivel hipofisario.

Diferentes investigaciones indicaron que la administración de melatonina o de ácido lipoico puede disminuir tanto la glucemia como los niveles séricos de colesterol y TAG ${ }^{52-55}$. Sin embargo, en nuestro estudio ninguno de los antioxidantes utilizados durante todo el período experimental afectó estos parámetros bioquímicos. Esta discrepancia podría deberse a diferencias en las rutas de administración, las dosis y la duración del tratamiento. 
Nuestros resultados muestran un aumento en los parámetros del daño oxidativo (niveles de lipoperóxidos y proteínas modificadas en nitro-tirosina), junto con la activación de la respuesta antioxidante (enzimas antioxidantes como catalasa y HO-1) en la glándula pituitaria de ratas tratadas con DRS. Ambos antioxidantes fueron eficaces en la prevención de los cambios en los parámetros del estrés oxidativo.

Por otra parte, el estrés oxidativo se asoció con la generación de un estado inflamatorio de bajo grado en el hígado y tejido adiposo (para una revisión, véase ${ }^{56,57}$ ). También se relacionó el aumento en el número de macrófagos del tejido adiposo con su expansión e inflamación, y con la aparición de IR y DM2. Sin embargo, la activación de macrófagos tisulares no se restringe al tejido adiposo, ya que también se ha demostrado en hígado, músculo esquelético e islotes pancreáticos ${ }^{58}$.

En particular, los resultados obtenidos en animales a los que se administró DRS durante tres semanas indican un aumento en el número de macrófagos hipofisarios y un incremento en los niveles de expresión de citoquinas inflamatorias, como TNF- $\alpha$ e IL1- $\beta$. Los macrófagos pueden producir reacciones inflamatorias no infecciosas a través del reconocimiento de patrones moleculares asociados al peligro (Danger Associated Molecular Patterns o DAMPs) liberados en una lesión celular. La unión de estos DAMP a receptores de reconocimiento de patrones activa diferentes vías intracelulares. Entre ellos, la vía del inflamasoma NLP3 que conduce a la producción de IL-1 $\beta$ e IL-18 ${ }^{59-61}$. Aunque se han detectado macrófagos tipo M1 y M2 en la hipófisis anterior de ratas controles ${ }^{62}$, el aumento en la producción de II-1 $\beta$ en estos animales sugiere una polarización a favor de macrófagos proinflamatorios.

El tratamiento con melatonina no sólo previno el aumento en el número de macrófagos, sino que también bloqueó la inducción de IL-1 $\beta$. En ese sentido estudios previos demostraron que la melatonina favorece el fenotipo antiinflamatorio M2 sobre M1 en células de Kupffer de hígado, macrófagos peritoneales y esplenocitos de ratones estresados ${ }^{63}$.

En cuanto a los efectos antiinflamatorios del ácido lipoico, también observamos una disminución en el número de macrófagos en la adenohipófisis que se asoció a una menor producción de TNF- $\alpha$. Estos resultados son similares a los obtenidos en tejido adiposo de ratones obesos ${ }^{64} \mathrm{y}$ en células mesangiales de riñón ${ }^{65}$.

Nuestra hipótesis sobre la participación de procesos oxidativos/inflamatorios en el desarrollo de la disfunción pituitaria parece ser consistente con la prevención inducida por la melatonina del aumento de los niveles hipofisarios y sistémicos de ACTH y corticosterona en ratas tratadas con DRS ${ }^{66}$. En ese sentido, estudios previos demostraron que la administración de melatonina (durante cinco días) redujo significativamente la secreción de corticosterona, atenuó la respuesta adrenocortical al estrés y aumentó la sensibilidad del eje HHA a la supresión por glucocorticoides $^{67,68}$. Resultados similares se obtuvieron en ratones con estrés crónico leve ${ }^{69}$ y en ratas diabéticas (estreptozotocina o STZ) tratadas con lipopolisacárido (LPS ${ }^{70}$. Más recientemente, Zhou et al. demostraron que la melatonina también disminuye los niveles de corticosterona en orina en ratas alimentadas con dietas de alto contenido de grasa bajo estrés crónico o en ratas diabéticas ${ }^{71,72}$.

La estimulación de la producción de ACTH hipofisaria (y de la corticosteronemia), por el ácido lipoico per se podría explicarse por un efecto directo del ácido lipoico sobre los corticotropos, como se demostró en la línea celular de ratón AtT20 estimulada con el ribonucleótido de 5-aminoimidazol-4-carboxamida $\left(\right.$ AICAR) ${ }^{73}$ o adiponectina ${ }^{74}$.

En resumen, nuestros resultados sugieren que la carga metabólica impuesta a la adenohipófisis por la administración de una dieta rica en sacarosa genera estrés oxidativo e inflamación en el tejido. Los DAMPs generados como consecuencia de los efectos nocivos del estrés oxidativo/nitrosativo sobre componentes celulares, atraen y estimulan macrófagos para producir citoquinas, que a su vez impactan en la producción de POMC por los corticotropos. Evidencias en favor de este mecanismo se obtuvieron recientemente en nuestro laboratorio ${ }^{66}$. No puede descartarse que la melatonina también bloquee la inhibición por ROS del efecto de los glucocorticoides sobre la producción de POMC, según lo descrito por Asaba et al. ${ }^{75}$.

Al prevenir la disfunción del eje HHA inducida por la dieta, la melatonina podría ser una opción terapéutica adecuada para atenuar los efectos metabólicos de la sobreproducción de glucocorticoides. Si bien en nuestras condiciones experimentales el tratamiento con melatonina no afectó la glucemia, la trigliceridemia o el peso corporal de los animales, se estudiaron efectos inhibitorios de la melatonina sobre la liberación de insulina en poblaciones de ratones con mutaciones específicas en el receptor 
MTNR1B de melatonina en islotes pancreáticos ${ }^{76}$. El uso efectivo de esta molécula en pacientes que presenten una disfunción del eje HHA deberá, por lo tanto, evaluarse clínicamente con estudios adicionales (que incluyen determinar la presencia o ausencia de polimorfismos específicos) sobre sus efectos metabólicos en cada uno de ellos.

\section{Agradecimientos}

Este estudio se realizó con fondos provenientes de subsidios recibidos del Consejo Nacional de Investigaciones Científicas y Técnicas (CONICET-PIP0257CO), la Sociedad Argentina de Diabetes (Área Básica, 2016), la Agencia Nacional de Promoción de Ciencia y Tecnología (ANPCyT-

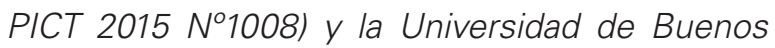
Aires (UBACYT-20020130100115BA). Los autores desean agradecer a la Dra. Carla Rothlin y al Dr. Souray Ghosh de la Universidad de Yale por su generosa contribución a este trabajo.

\section{BIBLIOGRAFÍA}

1. Eckel RH, Grundy SM, Zimmet PZ. SeminarThe metabolic syndrome; 2005.

2. Zimmet $P$, Alberti KG, Shaw J. Global and societal implications of the diabetes epidemic. Nature 2001; 414(6865):782-787.

3. Lê KA, Tappy L. Metabolic effects of fructose. Curr Opin Clin Nutr Metab Care 2006 Jul; 9(4):469-75. Doi:10.1097/01. mco.0000232910.61612.4d.

4. Dornas WC, de Lima WG, Pedrosa ML, Silva ME. Health implications of high-fructose intake and current research. Adv Nutr 2015; 6(6):729-737.

5. Dekker MJ, Su Q, Baker C, Rutledge AC, Adeli K. Fructose: a highly lipogenic nutrient implicated in insulin resistance, hepatic steatosis, and the metabolic syndrome. Am J Physiol Endocrinol Metab 2010; 299(5):E685-94.

6. Selenscig D, Rossi A, Chicco A, Lombardo YB. Increased leptin storage with altered leptin secretion from adipocytes of rats with sucrose-induced dyslipidemia and insulin resistance: effect of dietary fish oil. Metabolism 2010. Doi:10.1016/j.metabol.2009.09.025.

7. Zago V, Lucero D, Macri E V, Cacciagiu L, Gamba CA, Miksztowicz V, Berg G, Wikinski R, Friedman S, Schreier L. Circulating very-lowdensity lipoprotein characteristics resulting from fatty liver in an insulin resistance rat model. Ann Nutr Metab 2014; 56(3):198-206.

8. Chicco A, D'Alessandro ME, Karabatas L, Pastorale C, Basabe JC, Lombardo YB. Muscle lipid metabolism and insulin secretion are altered in insulin-resistant rats fed a high sucrose diet. J Nutr 2003; 133(1):127-133.

9. Arnaldi G, Scandali VM, Trementino L, Cardinaletti M, Appolloni G, Boscaro M. Pathophysiology of dyslipidemia in Cushing's syndrome. Neuroendocrinology 2010; 92 Suppl 1:86-90.

10. Peeke PM, Chrousos GP. Hypercortisolism and obesity. Ann NY Acad Sci 1995; 771:665- 676.

11. Pasquali R, Vicennati V, Cacciari M, Pagotto U. The hypothalamic-pituitary-adrenal axis activity in obesity and the metabolic syndrome. Ann NY Acad Sci 2006; 1083(1):111-28.
12. Brindley DN. Role of glucocorticoids and fatty acids in the impairment of lipid metabolism observed in the metabolic syndrome. Int J Obes Relat Metab Disord 1995 May; 19 Suppl 1:S69-75.

13. Paredes S, Ribeiro L. Cortisol: The villain in metabolic syndrome? Rev Assoc Med Bras (1992). 2014 Jan-Feb; 60(1):84-92. Doi:10.1590/1806-9282.60.01.017.

14. Pasquali $R$, Vicennati $V$, Cacciari M, Pagotto $U$. The hypothalamic-pituitary-adrenal axis activity in obesity and the metabolic syndrome. Ann NY Acad Sci 2006; 1083:111-128.

15. Tannenbaum BM, Brindley DN, Tannenbaum GS, Dallman MF, McArthur MD, Meaney MJ. High-fat feeding alters both basal and stress-induced hypothalamic- pituitary-adrenal activity in the rat. Am J Physiol Endocrinol Metab 1997; 273(6). Doi:10.1152/ajpendo.1997.273.6.e1168.

16. Martínez-Calejman C, Di Gruccio JM, Mercau ME, Repetto EM, Astort F, Sánchez R, Pandolfi M, Berg G, Schreier L, Arias P, Cymeryng $C B$. Insulin sensitization with a peroxisome proliferator-activated receptor $\gamma$ agonist prevents adrenocortical lipid infiltration and secretory changes induced by a high-sucrose diet. J Endocrinol 2012; 214(3):267-76.

17. Mercau ME, Repetto EM, Pérez MN, Martínez-Calejman C, Sánchez-Puch S, Finkielstein CV, Cymeryng CB. Moderate exercise prevents functional remodeling of the anterior pituitary gland in diet-induced insulin resistance in rats: role of oxidative stress and autophagy. Endocrinology 2016;157(3):1135-1145.

18. Martínez-Calejman C, Di Gruccio JM, Mercau ME, Repetto EM, Astort F, Sánchez R, Pandolfi M, Berg G, Schreier L, Arias P, Cymeryng $C B$. Insulin sensitization with a peroxisome proliferator-activated receptor gamma agonist prevents adrenocortical lipid infiltration and secretory changes induced by a high-sucrose diet. J Endocrinol 2012; 214(3):267-276.

19. Arkan MC, Hevener AL, Greten FR, Maeda S, Li ZW, Long JM, Wynshaw-Boris A, Poli G, Olefsky J, Karin M. IKK- $\beta$ links inflammation to obesity-induced insulin resistance. Nat Med 2005; 11(2). Doi:10.1038/nm1185.

20. Weisberg SP, McCann D, Desai M, Rosenbaum M, Leibel RL, Ferrante AW. Obesity is associated with macrophage accumulation in adipose tissue. J Clin Invest 2003; 112(12). Doi:10.1172/ JCl200319246.

21. Xu H, Barnes GT, Yang Q, Tan G, Yang D, Chou CJ, Sole J, Nichols A, Ross JS, Tartaglia LA, Chen H. Chronic inflammation in fat plays a crucial role in the development of obesity-related insulin resistance. J Clin Invest 2003; 112(12):1821-1830.

22. Bloch-Damti A, Bashan N. Proposed mechanisms for the induction of insulin resistance by oxidative stress. Antioxid Redox Signal 2005; 7(11-12):1553-1567.

23. Päth G, Scherbaum WA, Bornstein SR. The role of interleukin-6 in the human adrenal gland. Eur J Clin Invest 2000; 30 suppl 3:91-5. Doi:10.1046/j.1365-2362.2000.0300s3091.x.

24. Villar SR, Ronco MT, Fernández-Bussy R, Roggero E, Lepletier A, Manarin R, Savino W, Pérez AR, Bottasso O. Tumor necrosis factor- $\alpha$ regulates glucocorticoid synthesis in the adrenal glands of trypanosoma cruzi acutely-infected mice. The role of TNF-R1. PLoS One 2013; 22,8(5). Doi:10.1371/journal.pone.0063814.

25. Katahira M, Iwasaki Y, Aoki Y, Oiso Y, Saito H. Cytokine regulation of the rat proopiomelanocortin gene expression in AtT-20 cells. Endocrinology 1998; 139(5). Doi:10.1210/endo.139.5.6005.

26. Kariagina A, Romanenko D, Ren SG, Chesnokova V. Hypothalamic-pituitary cytokine network. Endocrinology 2004; 145(1):104-12. Doi:10.1210/en.2003-0669. 
27. Chesnokova V, Melmed S, Angeles CL, Angeles L. Minireview: neuro-immuno-endocrine modulation of the hypothalamicpituitary-adrenal (HPA) axis by gp130 signaling molecules. Endocrinology 2002; 143(5):1571-4.

28. Tkachenko I V, Jaaskelainen T, Jaaskelainen J, Palvimo JJ, Voutilainen $\mathrm{R}$. Interleukins 1 alpha and 1 beta as regulators of steroidogenesis in human $\mathrm{NCl}-\mathrm{H} 295 \mathrm{R}$ adrenocortical cells. Steroids 2011; 76(10-11):1103-1115.

29. Asaba K, Iwasaki Y, Asai M, Yoshida M, Nigawara T, Kambayashi M, Hashimoto K. High glucose activates pituitary proopiomelanocortin gene expression: possible role of free radicalsensitive transcription factors. Diabetes Metab Res Rev 2007; 23(4):317:23. Doi:10.1002/dmrr.677.

30. Takayasu S, Iwasaki Y, Nigawara T, Asai M, Yoshida M, Kageyama K, Suda T. Involvement of nuclear factor- $\mathrm{KB}$ and nurr-1 in cytokine-induced transcription of proopiomelanocortin gene in AtT20 corticotroph cells. Neuroimmunomodulation 2010; 17(2):88-96. Doi:10.1159/000258691.

31. Golbidi S, Badran M, Laher I. Diabetes and alpha lipoic acid. Front Pharmacol 2011; 2:69. Doi:10.3389/fphar.2011.00069.

32. Uskokovic A, Dinic S, Grdovic N, Jovanovic JA, Vidakovic M, Poznanovic G, Mihailovic M. Beneficial effects of $\alpha$-lipoic acid in diabetes- and drug-induced liver injury. Arch Biol Sci 2018. Doi:10.2298/ABS180503023U.

33. Flora SJS, Shrivastava R, Mittal M. Chemistry and pharmacological properties of some natural and synthetic antioxidants for heavy metal toxicity. Curr Med Chem 2013; 20(36):4540-74. Doi:10.2174/09298673113209990146.

34. Packer L, Kraemer K, Rimbach G. Molecular aspects of lipoic acid in the prevention of diabetes complications. Nutrition 2001; (10):888-95. Doi:10.1016/S0899-9007(01)00658-X.

35. Tordjman S, Chokron S, Delorme R, Charrier A, Bellissant E, Jaafari N, Fougerou C. Melatonin: pharmacology, functions and therapeutic benefits. Curr Neuropharmacol 2017; 15(3):434-443.

36. Reiter RJ, Tan DX, Manchester LC, Paredes SD, Mayo JC, Sainz RM. Melatonin and reproduction revisited. Biol Reprod 2009; 81(3):445-456.

37. Zhang HM, Zhang Y. Melatonin: a well-documented antioxidant with conditional pro- oxidant actions. J Pineal Res 2014; 57(2):131-46. Doi:10.1111/jpi.12162.

38. Reiter RJ, Tan D-X, Galano A. Melatonin reduces lipid peroxidation and membrane viscosity. Front Physiol 2014. Doi:10.3389/ fphys.2014.00377.

39. Reiter RJ, Mayo JC, Tan DX, Sainz RM, Alatorre-Jiménez M, Qin L. Melatonin as an antioxidant: under promises but over delivers. J Pineal Res 2016; 61(3):253-278.

40. Sharafati-Chaleshtori R, Shirzad H, Rafieian-Kopaei M, Soltani A. Melatonin and human mitochondrial diseases. J Res Med Sci 2017. Doi:10.4103/1735-1995.199092.

41. Favero G, Franceschetti L, Bonomini F, Rodella LF, Rezzani R. Melatonin as an anti-inflammatory agent modulating inflammasome activation. Int J Endocrinol 2017; 2017:1835195.

42. Korkmaz A, Reiter RJ, Topal T, Manchester LC, Oter S, Tan DX. Melatonin: an established antioxidant worthy of use in clinical trials. Mol Med 2009;15(1-2):43-50.

43. Salido EM, Bordone M, De Laurentiis A, Chianelli M, Keller Sarmiento MI, Dorfman D, Rosenstein RE. Therapeutic efficacy of melatonin in reducing retinal damage in an experimental model of early type 2 diabetes in rats. J Pineal Res 2013; 54(2):179:89. Doi:10.1111/jpi.12008.

44. Cymeryng CB, Dada LA, Podesta E. Effect of nitric oxide on rat adrenal zona fasciculata steroidogenesis. J Endocrinol 1998; 158(2):197-203.
45. Ohkawa H, Ohishi N, Yagi K. Assay for lipid peroxides in animal tissues by thiobarbituric acid reaction. Anal Biochem 1979; 95(2):351-8. Doi:10.1016/0003-2697(79)90738-3.

46. Aebi H. Catalase in vitro. Methods Enzymol 1984; 105:121-6. Doi:10.1016/S0076-6879(84)05016-3.

47. Mercau ME, Astort F, Giordanino EF, Martínez-Calejman C, Sánchez R, Caldareri L, Repetto EM, Coso OA, Cymeryng CB. Involvement of PI3K/Akt and p38 MAPK in the induction of COX-2 expression by bacterial lipopolysaccharide in murine adrenocortical cells. Mol Cell Endocrinol 2014; 384(1-2):43-51.

48. Livak KJ, Schmittgen TD. Analysis of relative gene expression data using real-time quantitative PCR and the 2 (Delta Delta C -T-) method. Methods 2001; 25(4):402-8. Doi:10.1006/ meth.2001.1262.

49. Dandona P, Aljada A, Chaudhuri A, Mohanty P, Garg R. Metabolic syndrome: a comprehensive perspective based on interactions between obesity, diabetes, and inflammation. Circulation 2005; 111(11):1448-1454.

50. Grattagliano I, Palmieri VO, Portincasa P, Moschetta A, Palasciano $\mathrm{G}$. Oxidative stress-induced risk factors associated with the metabolic syndrome: a unifying hypothesis. J Nutr Biochem 2008;19(8):491-504.

51. Fisher-Wellman K, Bloomer RJ. Macronutrient specific postprandial oxidative stress: relevance to the development of insulin resistance. Curr Diabetes Rev 2009; 5(4):228-238.

52. Sartori C, Dessen P, Mathieu C, Monney A, Bloch J, Nicod P, Scherrer $U$, Duplain $\mathrm{H}$. Melatonin improves glucose homeostasis and endothelial vascular function in high-fat diet-fed insulin-resistant mice. Endocrinology 2009; 150(12):5311-5317.

53. Cano-Barquilla $P$, Pagano ES, Jiménez-Ortega $V$, FernándezMateos P, Esquifino Al, Cardinali DP. Melatonin normalizes clinical and biochemical parameters of mild inflammation in diet-induced metabolic syndrome in rats. J Pineal Res 2014; 57(3):280-290.

54. Kitagawa A, Ohta Y, Ohashi K. Melatonin improves metabolic syndrome induced by high fructose intake in rats. J Pineal Res 2012; 52(4):403-413.

55. Maiztegui B, Román CL, Gagliardino JJ, Flores LE. Impaired endocrine-metabolic homeostasis: underlying mechanism of its induction by unbalanced diet. Clin Sci 2018; 132(8):869-881. Doi:10.1042/cs20171616.

56. Matulewicz N, Karczewska-Kupczewska M. Insulin resistance and chronic inflammation. Postep Hig Med Dosw 2016; 70(0):1245-1258.

57. Monteiro R, Azevedo I. Chronic inflammation in obesity and the metabolic syndrome. Mediat. Inflamm 2010;2010. Disponible en: http://www.ncbi.nlm.nih.gov/entrez/query.fcgi?cmd=Re trieve\&db=PubMed\&dopt=Citation\&l ist_uids=20706689.

58. Meshkani R, Vakili S.Tissue resident macrophages: key players in the pathogenesis of type 2 diabetes and its complications. Clin Chim Act 2016; 462:77-89.

59. Benetti E, Chiazza F, Patel NS, Collino M. The NLRP3 inflammasome as a novel player of the intercellular crosstalk in metabolic disorders. Mediat Inflamm 2006; 2013:678627.

60. HeY, Hara H, Núñez G. Mechanism and regulation of NLRP3 inflammasome activation. Trends Biochem Sci 2013; 41(12):1012-1021.

61. Netea MG, van de Veerdonk FL, Kullberg BJ, Van der Meer JWM, Joosten LAB. The role of NLRs andTLRs in the activation of the inflammasome. Expert Opin Biol Ther 2008; 8(12):186772. Doi:10.1517/14712590802494212.

62. Fujiwara K, Yatabe M, Tofrizal A, Jindatip D, Yashiro T, Nagai R. Identification of M2 macrophages in anterior pituitary glands of normal rats and rats with estrogen-induced prolactinoma. CellTissue Res 2017; 368(2):371-378. 
63. YiWJ, Kim TS. Melatonin protects mice against stress-induced inflammation through enhancement of M2 macrophage polarization. Int Immunopharmacol 2017; 48:146-158.

64. Deiuliis JA, Kampfrath T,Ying Z, Maiseyeu A, Rajagopalan S. Lipoic acid attenuates innate immune infiltration and activation in the visceral adipose tissue of obese insulin resistant mice. Lipids 2011; 46(11):1021-32. Doi:10.1007/s11745-011-3603-8.

65. Li G, Fu J, ZhaoY, Ji K, Luan T, Zang B. Alpha-lipoic acid exerts anti-inflammatory effects on lipopolysaccharide-stimulated rat mesangial cells via inhibition of nuclear factor kappa $B$ (NF-кB) signaling pathway. Inflammation 2015; 38(2):510-9. Doi:10.1007/s10753-014- 9957-3.

66. Mercau ME, Calanni JS, Aranda ML, Caldareri LJ, Rosenstein RE, Repetto EM, Cymeryng CB. Melatonin prevents early pituitary dysfunction induced by sucrose-rich diets. J Pineal Res 2019; 66(2):e12545.

67. Konakchieva R, Mitev Y, Almeida OF, Patchev VK. Chronic melatonin treatment counteracts glucocorticoid-induced dysregulation of the hypothalamic-pituitary-adrenal axis in the rat. Neuroendocrinology 1998; 67(3):171-180.

68. Konakchieva R, MitevY, Almeida OFX, Patchev VK. Chronic melatonin treatment and the hypathalamo-pituitary-adrenal axis in the rat: Attenuation of the secretory response to stress and effects on hypothalamic neuropeptide content and release. Biol. Cell 1997. Doi:10.1016/S0248-4900(98)80163-9.

69. Detanico BC, Piato AL, Freitas JJ, Lhullier FL, Hidalgo MP, Caumo W, Elisabetsky E. Antidepressant-like effects of melatonin in the mouse chronic mild stress model. Eur J Pharmacol 2009; 607(1-3):121-5. Doi:10.1016/j.ejphar.2009.02.037.
70. Zhong LY,Yang ZH, Li XR, Wang H, Li L. Protective effects of melatonin against the damages of neuroendocrine-immune induced by lipopolysaccharide in diabetic rats. Exp Clin Endocrinol Diabetes 2009; 117(9):463-469.

71. Zhou J, Wang D, Luo X, Jia X, Li M, Laudon M, Zhang R, Jia Z. Melatonin receptor agonist piromelatine ameliorates impaired glucose metabolism in chronically stressed rats fed a high-fat diet. J Pharmacol ExpTher 2018; 364(1):55-69.

72. Zhou J, Zhang J, Luo X, Li M, Yue Y, Laudon M, Jia Z, Zhang R. Neu-P11, a novel MT1/MT2 agonist, reverses diabetes by suppressing the hypothalamic-pituitary-adrenal axis in rats. Eur $\mathrm{J}$ Pharmacol 2017; 812:225-233.

73. Iwasaki Y, Nishiyama M, Taguchi T, Kambayashi M, Asai M, Yoshida M, Nigawara T, Hashimoto K. Activation of AMP-activated protein kinase stimulates proopiomelanocortin gene transcription in AtT20 corticotroph cells. Am J Physiol Metab 2007; 292(6). Doi:10.1152/ajpendo.00116.2006.

74. Chen M, Wang Z, Zhan M, Liu R, Nie A, Wang J, Ning G, Ma Q. Adiponectin regulates ACTH secretion and the HPAA in an AMPK-dependent manner in pituitary corticotroph cells. Mol Cell Endocrinol 2014; 383(1-2):118-25. Doi:10.1016/j.mce.2013.12.007.

75. Asaba K, Iwasaki Y, Yoshida M, Asai M, Oiso Y, Murohara T, Hashimoto K. Attenuation by reactive oxygen species of glucocorticoid suppression on proopiomelanocortin gene expression in pituitary corticotroph cells. Endocrinology 2004; 145(1):39-42.

76. Tuomi T, Nagorny CLF, Singh P, Bennet H, Yu Q, Alenkvist I, Isomaa B, Östman B, Söderström J, Pesonen A-K, Martikainen S, Räikkönen K, Forsén T, Hakaste L, Almgren P, Storm P, Asplund O, Shcherbina L, Fex M, Fadista J, Tengholm A, Wierup N, Groop L, Mulder H. Increased Melatonin Signaling Is a Risk Factor forType 2 Diabetes. Cell Metab 2016; 23(6):1067-1077. 